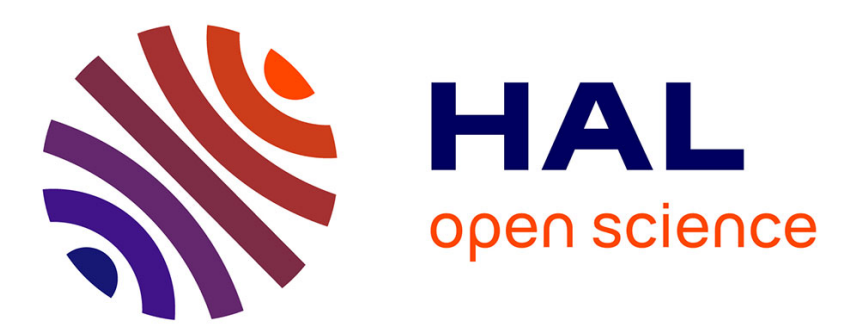

\title{
"Je ne me suis pas perdue et ne souhaite pas que l'on me trouve ". Stratégies des femmes dans les musées de sciences en Californie (xixe-
}

\author{
Leslie Madsen-Brooks
}

\section{- To cite this version:}

Leslie Madsen-Brooks. "Je ne me suis pas perdue et ne souhaite pas que l'on me trouve ". Stratégies des femmes dans les musées de sciences en Californie (xixe-. Culture et Musées, 2017, 30, pp.111-131. 10.4000/culturemusees.1229 . hal-03262013

\section{HAL Id: hal-03262013 \\ https://hal.science/hal-03262013}

Submitted on 16 Jun 2021

HAL is a multi-disciplinary open access archive for the deposit and dissemination of scientific research documents, whether they are published or not. The documents may come from teaching and research institutions in France or abroad, or from public or private research centers.
L'archive ouverte pluridisciplinaire HAL, est destinée au dépôt et à la diffusion de documents scientifiques de niveau recherche, publiés ou non, émanant des établissements d'enseignement et de recherche français ou étrangers, des laboratoires publics ou privés. 


\section{OpenEdition} Journals

\section{Culture \& Musées}

Muséologie et recherches sur la culture

$30 \mid 2017$

Musées au prisme du genre

\section{" Je ne me suis pas perdue et ne souhaite pas que l'on me trouve ". Stratégies des femmes dans les musées de sciences en Californie ( $x x^{e}-x x^{e}$ siècles)}

"I'm not lost and I won't be found". Strategies for success among 19th and 20th century women museum scientists in California

« No me perdí y no deseo que me encuentren ». Estrategias de las mujeres en los museos de ciencias en California $\left(x I x-x x^{e}\right)$

\section{LeSLIE MADSEN-BROOKS}

p. 111-131

https://doi.org/10.4000/culturemusees.1229

\section{Résumés}

Français English Español

Durant le dernier quart du xixe siècle et la première moitié du xxe, les femmes scientifiques en Californie n'ont pas seulement contribué aux efforts des musées d'histoire naturelle, aux herbariums, aux jardins, aux parcs zoologiques et à des organisations connexes mais en ont aussi fondé, transformé ou fait progressé de manière significative. Cet article examine les stratégies utilisées par plusieurs de ces chercheuses dans les musées, en mettant en exergue ce qui est commun à leur expérience - notamment leur déploiement de la synthèse muséale et leur subversion des attentes liées au genre. Cet article explore également les raisons pour lesquelles le contexte californien s'est révélé si favorable pour les femmes dans le domaine de l'histoire naturelle. Parmi celles-ci, on citera la diversité des biomes, l'accueil précoce par les hommes de la communauté scientifique qui avaient besoin d'aide pour cataloguer les espèces de l'État, une richesse employée de manière stratégique, le système routier ainsi que l'esprit d'initiative et l'entrepreneuriat combatif de ces femmes. 
In the third quarter of the nineteenth century and first half of the twentieth century; women scientists in California not only joined the efforts of natural history museums, herbaria, gardens, zoos, and related organizations, but also founded, significantly advanced, or transformed them. This article examines the strategies used by several such women museum scientists, highlighting key commonalities in their experiences - notably their deployment of the museum synthesis and their subversion of gendered expectations. The article also explores reasons why the California context proved so fruitful for women natural scientists. Among these are its diverse biomes, an early welcome by scientific men seeking assistance in cataloguing the state's species, strategically applied wealth, its roadways, and a spirit of initiative-taking and women's scrappy entrepreneurialism.

Durante el último cuarto del siglo XIX y la primera mitad del siglo XX, las mujeres científicas en California han contribuido con los esfuerzos de los museos de historia natural, herbariums, jardines, parques zoológicos y de organizaciones conexas, y también les han fundado, transformado o hecho avanzar de manera significativa. Este artículo examina las estrategias utilizadas por varias de estas investigadoras en los museos, destacando los puntos comunes de sus experiencias, sobretodo en cuanto al despliegue de la síntesis museística y su subversión de las expectativas vinculadas al género. Este artículo explora igualmente las razones por las cuales el contexto californiano es tan favorable para las mujeres en el dominio de la historia natural. Entre éstas podemos citar la diversidad de biomas, la acogida precoz por parte de los hombres de la comunidad científica, quienes necesitaban ayuda para catalogar las especies del Estado, y cuya riqueza fue empleada de manera estratégica, del sistema de rutas al espíritu de iniciativa y empresarial combativo de estas mujeres.

\section{Entrées d'index}

Mots-clés : femmes en sciences, Californie, histoire des musées, botanique, jardins

Keywords: women in science, California, museum history, botany, gardens

Palabras clave: mujeres en ciencias, California, historia de los museos, botánica, jardines

\section{Notes de la rédaction}

Manuscrit reçu le : 12 décembre 2016

Version révisée reçue le : 6 juin 2017

Article accepté pour publication le : 27 juin 2017

\section{Texte intégral}

$1 \quad$ En 1853, un groupe de sept scientifiques amateurs, tous des hommes, fondent l'Académie des sciences de Californie afin de promouvoir le développement et la diffusion des connaissances en sciences naturelles (Smith, 1987 : 114). À cette époque, même un homme avait des difficultés pour se former en sciences sans quitter les États-Unis, et la Californie était loin de disposer des mêmes ressources scientifiques que la côte est.

2 En parallèle, la biodiversité très riche de la Californie et la taille relativement restreinte de la population qui s'y était implantée rendaient difficile le vaste chantier que représentait la création de l'Académie des sciences de Californie. Les fondateurs s'accordèrent donc pour accueillir des femmes aussi bien dans le cadre d'une recherche fondamentale que pratique, sur le terrain : « Nous approuvons grandement l'aide des femmes dans tous les domaines des sciences naturelles et nous les invitons à coopérer. » (Leviton \& Aldrich, 1997 : 53.) L'Académie des sciences de Californie devenait alors rapidement la première institution scientifique importante de l'État. Ses fondateurs et premiers membres adoptaient les signes extérieurs d'une institution d'histoire naturelle de premier ordre : une bibliothèque, des collections de spécimens, des conférences, et par la suite des expositions.

3 Les femmes ayant émigré vers la Californie au cours des cinquante premières années de son existence en tant qu'État avaient leurs propres moyens de subsistance ou savaient comment profiter de la ruée vers l'or et des possibilités offertes par la région (Levy, 1992 : 92). Cette combinaison d'opportunités, de ressources et d'entrepreneuriat leur permit non seulement d'émerger en tant que participantes mais aussi d'occuper des postes importants dans les milieux de l'histoire naturelle. En Californie, les femmes ont fondé, financé, 
sauvé, maintenu et développé des collections scientifiques significatives ; elles ont bâti leurs propres institutions ou les ont soutenues financièrement en montant des affaires ; elles ont contribué à la compréhension professionnelle et publique des sciences naturelles ; et elles ont su gagner le respect de leurs homologues masculins ainsi que la reconnaissance et l'admiration du grand public. Elles ont concouru à la création d'un large éventail d'institutions d'histoire naturelle, dont un institut de recherche océanographique, un parc zoologique, des herbiers, un jardin botanique et des musées universitaires.

$4 \quad$ Les différentes institutions scientifiques californiennes ne ressemblaient pas à celles de la côte est. Bien que cette région ait été visitée pour la première fois par les Européens en 1542 et que ces derniers s'y soient établis en 1769, sa colonisation n'a été rapide qu'après la ruée vers l'or qui a commencé en 1848. Alors que des scientifiques amateurs et professionnels travaillaient déjà depuis un certain temps au catalogage du tiers est des États-Unis, la Californie vit arriver tardivement des chercheurs en sciences naturelles. Devant le manque criant de ressources universitaires, de contacts influents, de laboratoires et de bibliothèques que possédait au contraire la côte est des États-Unis, et en l'absence de fonds budgétaires pour les construire, les scientifiques de Californie travaillèrent de manière plus empirique, en créant des laboratoires marins, des arboretums, des jardins botaniques plutôt que des musées. La vénérable Académie des sciences de Californie elle-même n'a connu sa première exposition publique que vingt ans après sa fondation (Cal Academy, 2016). Néanmoins, les institutions que les Californiens créaient, dotaient de personnel ou soutenaient activement, se conformaient aux pratiques muséales traditionnelles : elles possédaient des collections bien cataloguées, des agendas de recherche scientifique, un personnel professionnel, et proposaient des expositions diverses, des horaires de visite pour le public ainsi que des programmes pédagogiques.

5 Chacune des femmes travaillant dans ces musées a pris un chemin différent vers la reconnaissance. Certaines ont utilisé leur fortune de manière stratégique, fondant des institutions et finançant des expéditions ; d'autres ont bâti des collections ; certaines se sont occupées de vulgariser les connaissances ; d'autres encore ont passé une grande partie de leur temps à recueillir et à préparer des spécimens. Certains académiciens pourraient penser que ces femmes n'étaient pas à proprement parler des professionnelles du musée, mais cet article les étudie en tant que telles car elles ont effectué des tâches comparables ou complémentaires pour la construction, la préservation et la diffusion des collections d'histoire naturelle. Elles ont toutes profité de la biodiversité californienne, d'une nature sauvage proche des zones urbaines (ainsi que du réseau grandissant de routes et de chemins de fer), de la relative richesse de cette région (et dans certains cas d'une fortune personnelle), ainsi que d'un public réceptif à leur travail. Ces chercheuses partageaient également de nombreuses caractéristiques : la plupart étaient indépendantes des hommes, par choix ou malgré elles, se sont révélées pleines de ressources et d'esprit d'initiative, et n'ont pas craint de faire connaître leurs opinions à des hommes plus puissants et davantage reconnus qu'elles. Afin de mieux comprendre les stratégies qui les ont amenées au succès dans les musées et leurs institutions connexes, cette recherche examine leur expérience selon un point de vue associant féminisme, synthèse muséale et performance de genre.

\section{L'histoire naturelle d'un point de vue féministe}

$6 \quad$ Même s'il s'agit de nouvelles organisations, les institutions d'histoire naturelle de Californie sont nées de traditions intellectuelles établies. Aux XVIIe et XIXe siècles, la reconnaissance de la complexité du monde naturel a engendré un nouveau type d'histoire - l'histoire naturelle (Foucault, 1971 : 128-130). Elle est différente de la pratique historique qui l'a précédée en ce sens qu'elle a émergé, du moins au début, non pas « d'autres mots, 
des textes ou des archives, mais d'espaces clairs où les choses se juxtaposent : des herbiers, des collections, des jardins » (Foucault, 1966 : 143). Ce nouvel espace - l'espace littéral de la collection ou du jardin, mais aussi l'espace intellectuel d'une discipline en émergence a généré de nouvelles manières de voir et de décrire les plantes et les animaux. Michel Foucault a reconnu que ces méthodes et perspectives émergentes rétrécissaient la focale des scientifiques ; l'observation scientifique consiste à « voir systématiquement peu de choses " dans l'espoir de donner aux choses « un nom que chacun pourra entendre » (ibid., p. 146). M. Foucault conçoit ce " chacun » de manière plus étroite que les philosophes des sciences féministes ou les chercheuses dans les musées considérées ici. Alors que M. Foucault se centre sur la manière dont l'histoire naturelle, en tant que discipline, est née de la myopie délibérée d’une communauté scientifique émergente, les femmes des musées californiens ont créé des institutions qui ont d'emblée été d'une grande rigueur scientifique et attentives à leur attrait potentiel auprès d'un large public.

M. Foucault note que les collections des institutions d'histoire naturelle sont des signifiants importants : " Leur importance, pour la culture classique, ne tient pas essentiellement à ce qu'ils permettent de voir, mais à ce qu'ils cachent et à ce que, par cette oblitération, ils laissent surgir. » (Ibid., p. 150.) Dans le dernier quart du XXe siècle, les philosophes féministes des sciences se sont demandé non pas quelles observations, mais de qui émanaient les observations, en notant que « les scientifiques [hommes] basés dans des institutions établissaient les principales manières de voir de la discipline, les méthodes hégémoniques et officielles d'appréhender les choses », alors que le type de sciences naturelles que pratiquaient les femmes, par exemple l'étude de la nature, étaient marginalisé par une science étasunienne en voie de professionnalisation rapide (Gates, 1998 : 87). Les philosophes se sont demandé comment les sciences naturelles ont permis aux femmes d'émerger en tant que productrices de savoirs et de discours - ou, inversement, comment elles leur ont rendu cette tâche difficile. Sandra Harding observe qu'au fur et à mesure que les femmes sont entrées dans ces espaces dominés par les hommes, elles ont forgé leurs propres espaces et ont commencé à critiquer la pratique scientifique régnante, considérant que " les choses ont un aspect différent si l'on commence à y penser du point de vue de notre vie » (Harding, 2008 : 114-115). Les philosophes jugeaient ces points de vue comme étant des «positionnements »; des positionnements qui se distinguent des perspectives du fait qu'ils sont acquis au prix d'une lutte menée contre les discours et les pratiques en place (Harding, 1991 : 127). Durant leur vie professionnelle, les Californiennes considérées dans cet article ont incarné ce phénomène du positionnement bien avant que cela ne soit explicitement affirmé par les philosophes des sciences.

8 La carrière de la directrice et fondatrice du parc zoologique de San Diego, Belle Benchley, qui n'avait pas reçu de formation en sciences - elle avait commencé comme gardienne de zoo en 1925 - et a géré sa ménagerie en se fondant sur son expérience de mère, est un premier exemple révélateur. Durant le quart de siècle où elle a dirigé le zoo de San Diego (1927-1953), B. Benchley a agi, comme elle le dit, en mère nourricière prenant soin de ses petits. Cherchant à améliorer la santé et la reproduction des animaux, elle a expérimenté différents types d'alimentation et d'habitats, et leur a prodigué affection et amour. Pour élargir le public du zoo à San Diego et au-delà, elle anthropomorphisait les animaux. Les titres de ses livres - My Life in a Man-Made Jungle [Ma vie dans une jungle façonnée par l'homme,] ; My Friends, the Apes [Mes amis les grands singes] ; et My Animal Babies [Mes bébés animaux], publiés respectivement en 1940, 1942 et 1945 révèlent sa manière de présenter le zoo et exposent sa politique d'administration aux visiteurs, donateurs potentiels ainsi qu'aux médias. B. Benchley mobilisait le vocabulaire de la famille pour transmettre ses découvertes à un public non averti. Elle ne rendait pas seulement les animaux sympathiques, mais elle présentait aussi la méthode scientifique comme une combinaison d'observation, d'expérimentation et d'intuition accessibles à toute personne susceptible de s'intéresser au monde animal. 
En public, B. Benchley gardait une attitude maternelle mais en coulisses, elle était une habile négociatrice, experte dans l'art de construire des réseaux. Elle a rapidement acquis des connaissances en matière de soin des animaux dans un parc zoologique, a embauché du personnel qualifié et prêt à s'adapter, et, malgré l'éloignement que représentait à l'époque la ville de San Diego, a su convaincre plusieurs autres directeurs de parcs zoologiques de son action. Dans les années 1940 et 1950, elle a par exemple correspondu avec William Mann, directeur du parc zoologique national de Washington. Les lettres qu'elle lui a envoyées le somment de l'aider à déjouer cet « honorable M. Paperasse » et contiennent des demandes de contacts de marchands d'animaux (Benchley, 1949:1). Elle œuvrait aussi pour que les spécimens soient mieux répartis entre les différents parcs zoologiques américains et a donné à W. Mann en premier le droit de refuser d'intégrer certains animaux (Benchley, $1947: 2$; Benchley, $1948: 1$ ). Elle cajolait et taquinait W. Mann tout en lui demandant des conseils et ce dernier semblait apprécier à la fois cette relation avec une directrice de zoo combative, isolée géographiquement et légèrement excentrique, et le fait de pouvoir la faire bénéficier de son expérience et de son statut.

Dans sa correspondance publique et privée, B. Benchley montre comment nombre de chercheuses se livraient à ce que Kathy Ferguson appelle des « subjectivités mobiles », des positionnements qui « traversent et suivent des axes de pouvoir, eux-mêmes en mouvement, sans toutefois les habiter totalement.

11 Elles sont relationnelles, produites par des rencontres et des contacts mouvants mais durables, sans en être pour autant tout à fait prisonnières » (Ferguson, $1993: 154)$. Les cyniques pourraient rejeter le personnage dont B. Benchley présente les livres comme artificiels, conçus pour un large public, alors qu'en réalité elle s'occupait de l'administration, négociait des animaux en coulisse et échangeait de bons mots avec W. Mann. Ces deux aspects de sa personnalité sont pourtant authentiques selon B. Benchley. Haraway argumente contre l'idée d'unifier l'identité de la scientifique : "Le moi divisé et contradictoire ", écrit-elle, " est le seul qui puisse interroger les positionnements et être responsable, le seul qui puisse composer et faire correspondre les conversations rationnelles et les rêves fantastiques qui changent l'Histoire. L'image privilégiée des épistémologies féministes c'est la division, pas l'être. » (Haraway, 2007 : 122.) «Diviser, plutôt qu'être, est l'image privilégiée pour les épistémologies féminines de la connaissance scientifique. » (Haraway, 1988 : 586) Cette division était particulièrement courante parmi les scientifiques des musées de Californie, dont les responsabilités couvraient de multiples secteurs des sphères publique et privée, et qui devaient trouver un équilibre entre le fait d'être des scientifiques professionnelles et des femmes, tout en évoluant dans un moment culturel où ces deux catégories étaient souvent perçues comme s'excluant mutuellement.

\section{La synthèse muséale}

En adoptant des subjectivités divisées et mobiles en public et en privé, en étendant les imaginaires culturels du rôle des femmes, en développant et en défendant leur propre expertise, les femmes des musées de Californie ont tiré parti de la " synthèse muséale ${ }^{1}$ ». Elles ont fait preuve d'une expertise scientifique traditionnellement masculine dans des contextes qui avaient précédemment peu ou mal accueilli les femmes, et elles l'ont fait de manière cohérente avec les attentes culturelles concernant les tâches et les caractéristiques féminines (Madsen-Brooks, 2013 : 27). Les Californiens n'étaient pas les seuls à utiliser la synthèse muséale à la fin du XIXe et au début du XXe siècle. Des femmes travaillaient dans diverses agences scientifiques fédérales au sein et autour de la Smithsonian Institution, mais également dans des institutions aussi différentes que le jardin botanique de New York, le laboratoire de biologie marine de Woods Hole (Massachusetts) et plusieurs autres académies des sciences, parfois de moindre importance (Parilla, 2012 : s.p. ; Warner \& Erin, 2002 ; Goldstein, 2008). En dehors des sciences naturelles, les femmes avaient contribué en grand nombre au développement florissant des musées d'art et des 
organisations de conservation historique aux États-Unis (Schwarzer, 2010 : 17-19). La présence des femmes de longue date dans l'enseignement, du cours préparatoire à la terminale, et leur nombre croissant parmi les bibliothécaires ont également développé la perception que les femmes pouvaient être des diffuseurs et des gardiens compétents de la connaissance, sans être nécessairement des créatrices (Brand, 1983 : 394, 397). La participation des femmes à ces entreprises de partage des connaissances leur a certainement permis de prendre part plus largement aux institutions d'histoire naturelle, si ce n'est d'y contribuer de manière conséquente.

13 La synthèse muséale a aidé les femmes à investir les milieux professionnels des sciences, et une fois entrées, à élargir la communauté de personnes dont le travail était jugé scientifique. Leur participation au discours scientifique dans des journaux, des lettres, des conférences, et de manière informelle avec leurs collègues, leur permettaient de comprendre les enjeux qui étaient soulevés. Au lieu d'élaborer des hiérarchies discursives de connaissances et de pratique scientifique, elles appliquaient ce que Haraway avait préconisé : elles élaboraient une «pratique d'objectivité qui privilégie la contestation, la déconstruction, la construction passionnée, les connexions en réseau et l'espoir d'une transformation des systèmes de connaissance et des façons de voir » (Situated Knowledges, 584-85/ anthologie p. 120). Le développement d'un jardin scientifique par Susana Bixby Bryant en est un bon exemple. L'établissement par S. B. Bryant du jardin botanique de Rancho Santa Ana a mis en exergue les tensions sur ce qui constituait une expertise scientifique dans les institutions d'histoire naturelle ainsi que le désir de ces institutions d'équilibrer le service rendu au public et à la communauté scientifique. L'action de S. B. Bryant et sa capacité à résoudre les tensions sont un exemple d'application habile de la synthèse muséale.

En mars 1926, S. B. Bryant informait Charles Singer Sargent de l'arboretum Arnold à l'université de Harvard, parce qu'on lui avait dit qu'il " s'intéressait tout particulièrement » à la flore californienne, qu'elle espérait " établir un jardin botanique pour faire pousser des arbres, des plantes et des fleurs indigènes » à Yorba Linda. " Sur mon ranch dans le canyon de Santa Ana, au pied des montagnes entre Los Angeles et Riverside, » écrit-elle, "j'espère développer un arboretum [...] et je souhaiterais planifier les choses de manière à ce que le projet puisse devenir une précieuse contribution pour d'autres passionnés de nos magnifiques plantes indigènes d'une incroyable diversité qui poussent en milieu sauvage à profusion et prolifèrent d'un bout à l'autre de notre État » (Bryant, 1926a : 1).

15 À la suite d'une visite de S. B. Bryant, C. S. Sargent lui écrivit pour la décourager d'établir un jardin d'une telle ampleur, de le limiter à 50 acres (un peu plus de 20 hectares, N.D.L.R.) - S. B. Bryant avait suggéré 165 - et de n'utiliser que des plantes qui poussent naturellement sur le site, sans irrigation. Il concluait ainsi sa lettre : « J'ai eu grand plaisir à vous voir ici et je regrette de ne pas avoir pu vous encourager dans ce projet qui vous tient tant à cœur. " (Sargent 1926 : 2.) S. B. Bryant répondit que son soutien pour un projet restreint et son « opposition sincère » à son projet plus ambitieux avaient renouvelé son inspiration de créer son jardin botanique. " D’une manière féminine toute traditionnelle, je vais faire un compromis en poursuivant mon projet initial » (Bryant, $1926 b$ : 1). Cet exemple illustre comment $S$. B. Bryant déploie la synthèse muséale ; elle reconnait que son obstination est un trait « féminin » et n'abandonne pas ses prétentions dans le cadre de l'expertise.

16 Ce bref échange de lettres entre S. B. Bryant et C. S. Sargent, ainsi qu'une autre série d'échanges entre elle et Willis Linn, révèlent que des botanistes professionnels doutaient qu'elle réussisse à établir un jardin vraiment scientifique. D’emblée, Jepson s'était montré très enthousiaste à propos du projet de S. B. Bryant car il pensait qu'une collection exhaustive de plantes indigènes vivantes utile aux botanistes manquait en Californie. Cependant, au fur et à mesure que le jardin grandissait en surface et en portée, Jepson commença à douter de sa gestion par la jeune femme. Il la percevait comme une donatrice de terres, d'argent et de ressources, mais non comme son homologue sur un plan 
intellectuel. Il refusa d'appuyer son travail en tant que directrice, écrivant qu'en qualité de femme d'affaires, elle demeurait ignorante « de l'éthique et des canons professionnels gouvernant les échanges entre botanistes ». Il lui reprochait de vouloir dupliquer des efforts déjà en cours dans des " pépinières, des parcs, des jardins d'exposition, des exploitations de semences, des jardins de fleurs sauvages et autres » (Jepson, $1929: 1-2)$.

S. B. Bryant n'apprécia guère cette " tirade de condamnation injustifiée de [s]es efforts », rappelant qu'elle voulait créer ce jardin surtout pour l'instruction et le plaisir du public (Bryant, 1929 : 1). Contrairement à Jepson, elle ne pensait pas que servir les scientifiques et accueillir le public étaient incompatibles. Pendant des décennies, les musées et les institutions d'histoire naturelle avaient satisfait ces deux populations. À Rancho Santa Ana, pour répondre aux besoins des chercheurs, l'herbarium du jardin fut catalogué conformément aux pratiques de Harvard, Stanford et de l'université de Californie, et S. B. Bryant avait rassemblé dès 1929 une bibliothèque de 1200 volumes (California Citrograph, 1929 : 263). Son personnel enregistrait l'origine de chaque graine, scion ou plante (California Citrograph, 1933 : 215). En 1930, elle embaucha Cari Wolf, titulaire d'un doctorat en botanique de Stanford, pour surveiller le développement scientifique du jardin (McCracken, 1933 : 13). En même temps, elle planifiait le jardin en tenant compte des "passionnés » de plantes indigènes; dans des lettres adressées à $\mathrm{C}$. S. Sargent et Jepson, elle se décrit comme une personne «follement passionnée » et dit qu'elle espère attirer d'autres «fous passionnés » dans son jardin scientifique. En outre, elle projetait de mettre à disposition des aires de pique-nique et de « donner des concerts à l'extérieur le dimanche après-midi destinés au "public" ! » (Bryant, 1926a : 1).

Ce faisant, la formulation du jardin par Jepson laissait à S. B. Bryant peu d'espace de participation. D'une part, elle était trop femme d'affaires et d'un statut social trop élevé pour être une véritable scientifique, et d'autre part, il ne voulait pas qu'elle et son jardin soient affiliés aux amateurs. Elle était par conséquent prise dans un un entre-deux entre la botanique professionnelle et celle pratiquée en amateur. Elle réussit cependant à s'échapper de cet espace par l'application minutieuse de la synthèse muséale. Elle acquit suffisamment de connaissances scientifiques pour créer un jardin scientifique dont le terrain et les résultats de recherche étaient accessibles au public tout en jouant le rôle d’une généreuse hôtesse.

19 Il est important de noter que, bien que la synthèse muséale ait aidé des femmes telles que S. B. Bryant à s'emparer de nouvelles opportunités, elle ne pouvait pas ouvrir toutes les portes ; dans certains contextes, les femmes restaient contraintes par les préjugés de genre. Si les musées et les universités organisaient régulièrement des expéditions de collecte pour les botanistes, il était difficile pour une femme célibataire d'accompagner des hommes à l'époque ; les femmes étaient donc confrontées à des obstacles supplémentaires pour collectionner. Dans une lettre datant de 1928, le naturaliste Vernon Bailey du département d'Agriculture des États-Unis écrivait à la collectionneuse Ynes Méxia : " Il n'est malheureusement pas possible que votre intérêt et votre enthousiasme trouvent le terrain approprié pour être pleinement utiles. J'aurais aimé vous aider si je le pouvais mais je vois des obstacles à laisser une femme participer à une expédition organisée par un musée. Vous auriez dû épouser le Dr Nelson et l'emmener avec vous. » (Bailey, 1928 : 1.) De même, la botaniste Agnes Chase du département d'Agriculture des États-Unis et du Smithsonian Institute écrivit à Y. Méxia que lorsqu'elle avait demandé à faire partie d'une expédition de collecte au Brésil, on lui avait répondu qu' "aucune disposition ne pouvait être prise pour une femme," et que par conséquent, si une dame d'âge mûr n'était déjà pas autorisée, il y avait peu de chances qu'elle le soit » (Chase, $1927: 1$ ). Leur solution à cette exclusion des expéditions par les hommes ? A. Chase et Y. Méxia, qui ne s'étaient jamais rencontrées en personne, financèrent ensemble une expédition au Brésil (Chase, 1928 : 2). 


\section{Le jeu du genre dans la synthèse muséale}

Le sexe et le genre sont des catégories confuses et controversées. Si les individus au XXIe siècle peuvent choisir parmi une diversité d'orientations de sexe et de genre, les Américains du XIXe et du début du XXe siècle n’avaient pas cette liberté. Les scientifiques des musées évoquées ici semblent avoir vécu avec une définition relativement étroite de ce que devait être culturellement et socialement, à l'époque, une femme. À certains moments cependant, chacune d'elles a repoussé les limites de ce qui était considéré comme approprié en transgressant les attentes sur ce qu'une femme devait savoir ou comment elle devait se comporter. Dans le cadre de la synthèse muséale, il existe un léger paradoxe : la formulation exprime un mélange de caractéristiques attendues et d'expertise inattendue. Ainsi, la seconde partie de la synthèse muséale menace toujours d'ébranler la première ; c'est un exercice d'équilibre délicat. Les femmes avaient beaucoup à gagner en élargissant le rôle traditionnel qui leur était assigné mais celles qui ne montraient pas les vertus de la condition féminine pouvaient perdre le respect de leurs collègues et les soutiens de certains musées.

Trois femmes illustrent bien cet équilibre et la nécessité de repousser les limites : les botanistes de l'Académie des sciences de Californie Katharine Brandegee et Alice Eastwood, et la collectionneuse de plantes indigènes Lester Rowntree. K. Brandegee, une jeune veuve arrivée à San Francisco en 1875 pour faire des études de médecine, est devenue conservatrice en botanique de l'Académie des sciences de Californie en 1883 (Weiss, 2014 : s.p.). La personnalité de K. Brandegee se manifeste dans certaines de ses publications scientifiques et transparaît dans les descriptions que d'autres personnes ont pu faire d'elle. Dans son histoire des naturalistes de San Francisco, Joseph Ewan écrit que son « deuxième mariage fut aussi heureux pour la botanique que pour le couple. Marcus Jones m'a un jour fait la remarque que "Brandegee aurait dû être né femme et Mme. Brandegee homme.

Donc leur mariage ne pouvait être qu'un succès !” » Que voulaient dire J. Ewan et M. Jones par ces remarques ? J. Ewan poursuit en décrivant T. S. Brandegee comme un " homme discret ", réticent, " modeste et sans prétentions » qui « pouvait parfois s'exprimer de manière vive » (Ewan, 1955 : 33). K. Brandegee, en revanche, avait un caractère tout à l'opposé. Dans un commentaire de 1892 sur la nomenclature botanique, elle interpelle des botanistes qui ont proposé des révisions de la taxonomie botanique qu'elle juge fausses ou inutiles. Elle insinue qu'un autre botaniste est narcissique, et elle utilise des adjectifs et des phrases comme « affreux », " inexcusable », « inadmissible », « illogique » et «qui nous est infligé » pour décrire le travail d'autres personnes (Brandegee, 1892 : 166, 171-172). Ce n'était pas quelqu'un d'enclin à épargner la sensibilité des gens - même lorsqu'il s'agissait d'hommes respectés dans son domaine - si elle pensait qu'ils avaient tort.

Alors que K. Brandegee cultivait un côté narquois et prenait parti, sa protégée Alice Eastwood faisait preuve d'une " gentillesse et d'une obligeance sans faille » (Chase, 1938 : 1). Les photographies d'A. Eastwood tout au long de sa vie la montrent souriante, portant des fleurs fraîchement coupées sur son chapeau, des robes courtes à l'encontre de la mode de l'époque et des bottes en cuir montant jusqu'au mollet. Son biographe, qui a eu la possibilité d'interviewer une A. Eastwood âgée mais encore intellectuellement vive, écrit que "l'injection de sa personnalité unique et non conventionnelle dans les cercles compassés de l'Académie était tonique », et que pendant les décennies qu'elle a passées à l'Académie, elle s'est trouvée entourée d'« hommes formant un cercle grandissant d'admirateurs » (Wilson, 1955 : 49, 64). Pourtant, l'esprit d'A. Eastwood restait cantonné à la botanique, sans se préoccuper du mariage, et de fait, elle utilisait sa carrière bien remplie comme excuse pour se lier d'amitié avec des collègues hommes mais en évitant 
tout lien amoureux : « Je ne pouvais tout simplement pas les laisser gêner mon travail. » (Wilson, $1955: 64$. )

24 La jeune A. Eastwood avait déjà gagné le respect des botanistes à travers les États-Unis, mais elle est devenue internationalement célèbre parmi les naturalistes et les scientifiques des musées quand, immédiatement après le tremblement de terre de San Francisco en 1906, cette femme de 47 ans se précipita à l'Académie des sciences de Californie et risqua sa vie pour sauver les spécimens botaniques (Eastwood, $1906: 824-26$; Wilson, 1955 : 9096). Plus de trois décennies plus tard, le jour de son 80 anniversaire, d'autres botanistes firent remarquer l'héroïsme qu'elle avait montré à une époque où les femmes botanistes au Smithsonian Institute n'avaient même pas le droit de faire partie de la société principale de botanique de Washington (Chase, 1938 : 1). Ces efforts étaient exceptionnels mais le fait qu'ils avaient été ceux d'une femme était à l'époque proprement étonnant.

Comme beaucoup de femmes de classe moyenne de sa génération, A. Eastwood était attirée par le travail d'éducation et de réforme, qui dans son cas prit la forme de la conservation, de l'éducation en botanique et du lobbying pour la préservation d'espaces ouverts naturels et urbains. Elle donnait des conférences dans des clubs, dirigeait des expéditions sur le terrain pour explorer la flore régionale et créa les premières expositions de fleurs fraîches à l'Académie. Au lieu de faire partie d'organisations scientifiques élitistes, elle s'investissait dans des groupes de botanique et des organisations de conservation ouverts au public ${ }^{2}$. Elle incitait les femmes des clubs à s'impliquer dans les questions locales ; lorsque des promoteurs menacèrent de construire dans le Golden Gate Park, elle rappela aux femmes du California Club à San Francisco le pouvoir de leur voix : " Le maire et ses collaborateurs n'oseront pas faire cela si on leur fait sentir que les femmes s'en souviendront et les puniront. » (Eastwood 1916 : 1.) Les résidents locaux appréciaient ses efforts et elle était très touchée de leur affection. Nonagénaire, A. Eastwood se vantait auprès d'A. Chase : « Ils m'appellent la botaniste du jardinier » et « le Club de jardinage des hommes d'affaires m'appelle leur chérie » (Eastwood 1949: 2).

En revanche, la botaniste Lester Rowntree, bien qu'elle ne fût pas l'opposée d'A. Eastwood, était une " solitaire » (James Roof, in Levenson, 1978 : 78). L. Rowntree, qui vécut jusqu'à l'âge de 100 ans, passa les cinquante premières années de sa vie d'une manière relativement conventionnelle, s'occupant de ses parents puis de son mari et de son fils. Néanmoins, elle rompit brusquement avec la tradition dans la seconde partie de sa vie. Comme l'explique l'historienne Rosemary Levenson, " à l'âge de 53 ans, Lester divorça de son mari et commença une nouvelle carrière, abandonnant le nom de Gertrude Ellen et adoptant le nom androgyne de Lester Rowntree. Lester n'avait pas apprécié le rôle féminin stéréotypé qu'elle avait été obligée de jouer » (Levenson, 1978 : iv). L. Rowntree lança sa carrière en tant que spécialiste des plantes indigènes une fois que - selon ses propres termes - « [s]on bonheur domestique a volé en éclats » en 1932 (Rowntree, 1939: 809). Dans une voiture dédiée à la collecte de plantes, elle parcourut seule les autoroutes et les routes secondaires de Californie, s'écartant des routes à pied dans la nature (L. B. Rowntree and R. A. Rowntree, 2006 : xxxiii ; Rowntree, 1947 : 94). Elle collectionnait des plantes sauvages pour les faire pousser dans son jardin, sur les hauteurs de Carmel, et pour les vendre à des jardiniers à travers le monde (Levenson, 1978 : 97). Elle entretenait une correspondance avec des conservateurs de musées, d'herbariums, de jardins botaniques et d'arboretums du monde entier, proposant de leur envoyer des spécimens ou leur demandant de l'aide pour identifier ce qu'elle avait trouvé (correspondance de Rowntree, 1926-1955).

27 Écrivaine de talent, L. Rowntree faisait l'apologie des qualités bénéfiques et de la beauté des plantes sauvages de l'État de Californie pour orner les jardins. Ses écrits botaniques, qui sont accessibles à tous, sont rendus plus intéressants par ses diverses anecdotes illustrant les défis qu'elle a rencontrés en tant que femme sur le terrain. Elle admet qu'elle a dû se battre pour maintenir l'équilibre de la synthèse muséale : " L'une des choses les plus difficiles dans ma vie est de combiner les projets d'une dame et ceux d'une romanichelle. Je ne veux abandonner ni l'une ni l'autre de ces formes d'existence, et 
pourtant elles ne sont vraiment pas compatibles. » (Rowntree 1939 : 810-11.) Elle était frustrée que certaines personnes viennent perturber cet exercice d'équilibriste, des personnes qui s'attendaient à une dame quand L. Rowntree voulait plutôt être une botaniste itinérante. Elle raconte, par exemple, une expédition de collecte où deux hommes cherchaient une femme égarée dans les montagnes et persistaient à dire qu'elle était perdue malgré ses protestations du contraire. Quand ils ont insisté pour qu'elle vienne avec eux, L. Rowntree a répondu : " Pas question. Je ne me suis pas perdue et ne souhaite pas que l'on me trouve. » (Rowntree, 1939 : 811.)

Ces femmes des musées conjuraient aussi régulièrement les attentes de genre ou celles concernant l'hétérosexualité des femmes. La plupart ont réussi dans leur vie professionnelle après s'être débarrassées des convenances domestiques ou maritales. S. B. Bryant a lancé son jardin une fois devenue veuve. B. Benchley et L. Rowntree ont chacune divorcé une fois, Y. Méxia deux fois - et toutes ont démarré leur carrière scientifique après leur divorce. Le mariage et le sexe avaient apparemment peu d'intérêt pour elles. A. Eastwood les évitait. Y. Méxia avait déménagé loin de son second mari avant leur divorce et elle a écrit qu'avoir des relations sexuelles avec lui ne l'intéressait pas, préférant vivre à ses côtés comme une sœur plutôt qu'en qualité de femme (Méxia, $1911: 1$ ). L. Rowntree a également admis qu'elle n'était " pas généreuse de son corps » dans son mariage (Levenson, 1978 : 134). Annie Montague Alexander, une collectionneuse de petits mammifères et d'oiseaux qui avait fondé deux musées à l'université de Californie, Berkeley, s'est investie dans une domesticité non conventionnelle avec une autre femme pendant quatre décennies. L. Rowntree adopta un nom d'homme (Stein, $2001:$ 117, 119). Devenue veuve, K. Brandegee s'employa à obtenir un diplôme de médecine et ses pairs l'ont décrite comme étant masculine.

Les remarques mordantes de K. Brandegee, l'énergie et la générosité sans bornes d’A. Eastwood, les diverses excentricités de L. Rowntree et l'humour de B. Benchley, tout comme leur expertise dans leurs domaines, les rendaient attachantes aux yeux de leurs pairs masculins. Et pourtant, si toutes profitèrent de ce que l'on attendait des femmes aux XIXe et XXe siècles en termes de capacité d'organisation des connaissances pour le public, elles ne rentraient pas dans le moule traditionnel de la femme ou de la mère. En tant que femmes de sciences, elles adoptèrent la synthèse muséale mais le firent dans le contexte californien avec un air entendu - une liberté que leurs consœurs scientifiques de la côte est pouvaient peut-être moins s'offrir comme à la Smithsonian Institution (MadsenBrooks, 2009 : 18-23). Leurs collègues masculins, notamment en Californie, semblaient apprécier cette subversion des normes de genre du moment qu'elle s'appuyait sur une profonde connaissance de leurs domaines. Ces femmes, ainsi que d'autres scientifiques californiennes, apportèrent un cachet nuancé à la synthèse muséale, en faisant jouer certaines caractéristiques " féminines ", en montrant une résistance à des croyances rétrogrades concernant les femmes et une certaine résilience face aux défis qui s'imposaient.

\section{Le contexte californien}

30 Il est facile de classer les musées de manière étroite, de n'y penser qu'en termes de bâtiments contenant des pièces exposées conservées par des experts. Cependant, comme le montre clairement l'expérience de ces femmes, les musées transcendent leurs bâtiments pour s'étendre dans un écosystème muséal plus vaste qui recouvre de nombreux types de travaux scientifiques moins visibles et évidents. Les opportunités qui se sont présentées aux femmes ont été à la fois générées et circonscrites par les normes culturelles et scientifiques de l'époque. La synthèse muséale - mélange d'expertise scientifique et d'attentes culturelles selon lesquelles les femmes font par exemple d'excellentes professeures et de très bonnes bibliothécaires, sont des éducatrices naturelles et restent plus proches de la nature - a donné aux femmes l'opportunité de participer 
professionnellement à l'histoire naturelle. En Californie notamment, il s'agissait de femmes qui, pour emprunter les termes de L. Rowntree, n'étaient pas perdues ; elles étaient bien là où elles devaient être et n'allaient pas croire les hommes qui leur disaient le contraire.

31 La Californie elle-même a également joué un rôle important dans le succès de ces femmes. Bien que cela n'ait pas été utopique pour les femmes, la communauté de sciences naturelles relativement progressiste de l'État acceptait une hétérogénéité et s'érigeait contre les règles strictes de la science plus directive de la côte est, par exemple avec ses classifications taxonomiques inébranlables. Alors que les scientifiques de la côte est bâtissaient des institutions bureaucratiques et montraient un certain intérêt pour les applications commerciales et gouvernementales des ressources naturelles, les scientifiques de Californie recherchaient ce que l'historien Michael Smith appelle « un modèle social moins rapace en faveur d'un usage humain de la nature dans le Far West » (Smith, 1987 : 185, 5-7, 58). Le jardin de S. B. Bryant, organisé comme le voulait la nature plutôt que selon les espèces, le soin maternel de B. Benchley vis-à-vis des animaux, l'entreprise de conservation des espèces indigènes de L. Rowntree et les expositions de fleurs sauvages d'A. Eastwood dans des jardinières bigarrées à l'Académie des sciences de Californie (car, disait-elle, elle " détest[ait] l'uniformité tout comme la nature la détest[ait] ») sont tous des exemples de ce phénomène où l'on commence et où l'on travaille avec le monde naturel plutôt que de le commercialiser (Eastwood, $1917: 1$ ).

32 La biodiversité exceptionnelle de l'État et son réseau de routes grandissant ont aussi ouvert aux femmes des possibilités de participation. L. Rowntree et A. Eastwood notamment profitèrent de l'infrastructure de l'État centrée sur la voiture. Elles ont également tiré profit d'un moment culturel aujourd'hui disparu où la civilisation commençait tout juste à étendre ses nombreux tentacules pavés dans les zones sauvages de l'État. Dans un récit des moments qu'il a passés à travailler sous la direction d'A. Eastwood, John Thomas Howell, son protégé et successeur, a résumé les dizaines d'expéditions sur le terrain avec elle, expéditions qu'elle finançait personnellement car son salaire ne lui permettait pas de les entreprendre. Leurs expéditions, dit-il, couvraient « une expérience du terrain si vaste que peu de collectionneurs de plantes l'ont égalée ou surpassée. Et maintenant que l'expansion des banlieues et les mauvaises herbes omniprésentes et étouffantes éliminent tant de notre flore des vallées et du pied des montagnes, je reste reconnaissant de la chance d'avoir pu aider à préserver dans des herbiers des traces de plantes et de leurs communautés que l'on ne trouve plus aujourd'hui » (Howell, $1975: 5$ ).

Le long récit de $\mathrm{J}$. T. Howell sur leurs nombreuses aventures aborde plusieurs thèmes qui ont souvent fait de la Californie un endroit extrêmement accueillant pour les femmes travaillant dans les sciences naturelles : cataloguer la biodiversité de l'État menacée par une population grandissante dépendait des efforts des scientifiques et du système d'autoroute en expansion qui a rendu des endroits autrefois éloignés plus accessibles, même pour des "dames botanistes " octogénaires ; les modestes parts de richesse de femmes, employées de manière stratégique, pouvaient avoir un impact considérable ; et les hommes botanistes de l'État ont réitéré leur admiration pour ces femmes scientifiques fortes et au franc parler. Alors que toutes étaient loin d'être les seules scientifiques de musée à avoir émergé en Californie au passage du XIXe au XXe siècle, les stratégies qu'elles ont déployées sont représentatives de leur temps, et constituent des modèles pour les générations ultérieures.

\section{Bibliographie}

Académie des sciences de Californie. 2016. " Our History ». Publication en ligne : <http://www.calacademy.org/our-history>. Consulté le 25 avril 2017. 
Bailey (Vernon) à Ynes Méxia. 18 avril 1928. Lettre. Documents Méxia (BANC 68/130m), bibliothèque Bancroft, boîte 1 , dossier 14 .

Benchley (Belle). 1945. My Animal Babies. Boston : Little, Brown and Company.

Benchley (Belle). 1942. My Friends, the Apes. Boston : Little, Brown and Company.

Benchley (Belle). 1940. My Life in a Man-Made Jungle. Boston : Little, Brown and Company.

Benchley (Belle) à William Mann. 28 février 1949. Archives de la Smithsonian Institution, unité de registres 74, boîte 174, dossier 2.

Benchley (Belle) à William Mann. 28 avril 1947. Archives de la Smithsonian Institution, unité de registres 74, boîte 174, dossier 2.

Benchley (Belle) à William Mann, 17 août 1948. Archives de la Smithsonian Institution, unité de registres 74, boîte 174, dossier 2.

Brand (Barbara). « Librarianship and Other Female-Intensive Professions ». The Journal of Library History, 18(4), p. 391-406.

Brandegee (Katharine). 1892. « The Nomenclature of Plants ». Zoe, 3, p. 166-72.

Bryant (Susana Bixby) à Charles Singer Sargent. 9 mars 1926. Lettre. Archives du jardin botanique de Rancho Santa Ana, dossier « Sargent, Charles Springer », lettre \#265B-8.

Bryant (Susana Bixby) à Charles Singer Sargent. 28 avril 1926. Lettre. Archives du jardin botanique de Rancho Santa Ana, dossier « Sargent, Charles Springer », lettre \#265B-6.

Bryant (Susana Bixby) à Willis Linn Jepson. 28 février 1929. Lettre. Archives du jardin botanique de Rancho Santa Ana, dossier « Willis Linn Jepson », pas de numéro de lettre.

Chase (Agnes) à Alice Eastwood. 29 décembre 1938. Archives de la Smithsonian Institution, unité de registres 229, division of Grasses, sér. 1 ; boîte 2.

Chase (Agnes) à Ynes Méxia. 13 décembre 1927. Documents Méxia (BANC 68/130m), bibliothèque Bancroft, université de Californie, Berkeley, carton 1, dossier 16.

Chase (Agnes) à Ynes Méxia. 9 mai 1928. Documents Méxia (BANC 68/130m), bibliothèque Bancroft, université de Californie, Berkeley, carton 1, dossier 16.

Eastwood (Alice). 1906. « L’Académie des sciences de Californie ». Science, 23(595), p. 824-826.

Eastwood (Alice) aux femmes du California Club à San Francisco. 10 février 1916. Documents Alice Eastwood, Academie des sciences de Californie, boîte 59, dossier " San Francisco ».

Eastwood (Alice) à Joseph Grinnell. 30 octobre 1917. Lettre. Archives de l'Académie des sciences de Californie, correspondance Eastwood, boîte 49.

Eastwood (Alice) à Agnes Chase. 1er janvier 1949. Archives de la Smithsonian Institution, unité de registres 229, division of Grasses, série 1, boîte 2 .

Ewan (Joseph). 1955. "San Francisco as a Mecca for Nineteenth-Century Naturalists », p. 1-63 in $A$ Century of Progress in the Natural Sciences. San Francisco : Académie des sciences de Californie.

Ferguson (Kathy). 1993. The Man Question : Visions of Subjectivity in Feminist Theory. Berkeley : University of California Press.

Foucault (Michel). 1971. The Order of Things : An Archaeology of the Human Sciences. New York : Pantheon Books. Réimpression (1994). New York : Vintage Books.

Gates (Barbara). 1998. Kindred Nature : Victorian and Edwardian Women Embrace the Living World. Chicago : The University of Chicago Press.

Goldstein (Daniel). 2008. " Outposts of Science : The Knowledge Trade and the Expansion of Scientific Community in Post - Civil War America ». Isis, 99, p. 519-546.

DOI : 10.1086/591711

Haraway (Donna). 2007. Manifeste cyborg et autres essais. SciencesFictions-Féminismes. Anthologie établie par Laurence Allard, Delphine Gardey \& Nathalie Magnan. Paris : Exils, collection « Essais ».

Haraway (Donna). 2004. "Modest_Witness@Second_Millennium », p. 223-250 in The Haraway Reader. New York : Routledge.

Haraway (Donna). 1988. "Situated Knowledges : The Science Question in Feminism and the Privilege of Partial Perspective ». Feminist Studies, 14(3), p. 575-599.

DOI : $10.2307 / 3178066$

Harding (Sandra). 2008. Sciences from Below :Feminisms, Postcolonialities, and Modernities. Durham, NC : Duke University Press.

Harding (Sandra). 1991. Whose Science ? Whose Knowledge ? Thinking from Women's Lives. Ithaca, NY : Cornell University Press. 
Howell (John Thomas). 4 avril 1975. " Alice Eastwood and John Thomas Howell », documents Eastwood, boîte 30 : Material About Her. Académie des sciences de Californie.

Jepson (Willis Linn) à Susana Bixby Bryant. 16 février 1929. Lettre. Archives du jardin botanique de Rancho Santa Ana, dossier « Willis Linn Jepson », pas de numéro de lettre.

Levenson (Rosemary). 1978. Lester Rowntree :California Native Plant Woman. Histoire orale. Berkeley, CA : Bureau régional d'histoire orale, bibliothèque Bancroft, université de Californie.

Leviton (Alan) \& Aldrich (Michele). (sous la dir. de). 1997. Theodore Henry Hittel's The California History of Sciences :A Narrative History, 1853-1906. San Francisco : Académie des sciences de Californie.

Levy (Joann). 1992. They Saw the Elephant : Women in the California Gold Rush Norman : University of Oklahoma Press.

Madsen-Brooks (Leslie). 2009. « Challenging Science as Usual : Women’s

Participation in Natural History Museum Work, 1870-1950 ». Journal of Women's History, 21(2), p. 11-38. Madsen-Brooks (Leslie). 2013. « A Synthesis of Expertise and Expectations : Women Museum Scientists, Club Women and Populist Natural Science in the United States, 18901950 ». Gender \& History, 25(1), p. 27-46.

McCracken (Floyd). 14 octobre 1933. « A Sanctuary for Wildings ». Los Angeles Times, p. 13.

Méxia (Ynes) à Manuel Reygadas. 21 novembre 1911. Lettre. Documents Méxia (BANC 68/130m), bibliothèque Bancroft, université de Californie, Berkeley, boîte 1, dossier 2.

s.n. 1933. « Mrs. Bryant Again Entertains Lemon Men’s Club at Field Day Meeting ». The California Citrograph, 13(8), p. 215.

Parilla (Lesley). 2012. "Women in Science, in the Field ». Field Book Project. Publication en ligne : <http://nmnh.typepad.com/fieldbooks/2012/o3/women-in-sciencein-the-field.html>. Consulté le 5 décembre 2016.

s.n. 1929. « Rancho Santa Ana Botanic Garden and Herbarium ». The California Citrograph, 14(6), p. 263.

Rowntree (Lester). 1936. Hardy Californians : A Woman's Life with Native Plants. Londres : MacMillan. Réimpression (2006). Oakland, CA : University of California Press.

Rowntree (Lester). 1939. « Lone Hunter ». The Atlantic Monthly (juin 1939) : 809-816, ici 809.

Rowntree (Lester). 1947. "On a Trail of Beauty », p. 93-130 in The Sierra Nevada :The Range of Light / sous la dir. de Roderick Peattie. New York : The Vanguard Press, Inc.

Rowntree (Lester). Plusieurs correspondances concernant la disposition de graines que Rowntree avait recueillies. Documents Lester Rowntree. Académie des sciences de Californie. 1926-1955. Boîtes 8, 9.

Rowntree (Lester B.) \& Rowntree (Rowan A.). 2006. " About Lester ", p. xv-xlvi in Hardy Californians : A Woman's Life with Native Plants par Lester Rowntree (1936). Oakland, CA : University of California Press.

Sargent (Charles Singer) à Susana Bixby Bryant. 17 avril 1926. Lettre. Archives du jardin botanique de Rancho Santa Ana, dossier « Sargent, Charles Springer », lettre \#265B-7.

Schwarzer (Marjorie). 2010. "Women in the Temple : Gender and Leadership in Museums », p. 1627 in Gender, Sexuality, and Museums / sous la dir. de Amy K. Levin. Londres : Routledge.

Smith (Michael). 1987. Pacific Visions : California Scientists and the Environment, 1850-1915. New Haven : Yale University Press.

Stein (Barbara). 2001. On Her Own Terms : Annie Montague Alexander and the Rise of Science in the American West. Oakland, CA : University of California Press.

s.n. « Échange avec M. [John Thomas] Howell - 25 avril 1967. » Note de l'archiviste. Documents Ynes Méxia, Académie des sciences de Californie, boîte 2.

Warner (Patricia Campbell) \& Erin (Margaret). 2002. "Wading in the Water : Women Aquatic Biologists Coping with Clothing, 1877-1945 ». BioScience, 52(1), p. 97-104.

Weiss (Amy). 2014. "Katharine Brandegee : Blazing a Trail for Women in Science ». New York Botanical Garden Science Talk Blog. Publication en ligne : <http://blogs.nybg.org/ sciencetalk/2014/02/katherine-brandegee-blazing-a-trail-for-women-inscience/>. Consulté le 22 novembre 2016.

Wilson (Carol Green). 1955. Alice Eastwood's Wonderland : The Adventures of a Botanist. San Francisco : Académie des sciences de Californie. 


\section{Notes}

1 C'est-à-dire la capacité du musée à redistribuer les rôles et à intégrer les compétences des femmes malgré les stéréotypes de genre. Nous avons choisi de traduire l'expression anglaise museum synthesis par « synthèse muséale » plutôt que « synthèse du musée » pour des raisons stylistiques (N.D.L.R.).

2 Les documents concernant A. Eastwood à l'Académie des sciences de Californie sont truffés de correspondances et/ou de cartes de membre de ces organisations.

\section{Pour citer cet article}

\section{Référence papier}

Leslie Madsen-Brooks, « « Je ne me suis pas perdue et ne souhaite pas que l'on me trouve ». Stratégies des femmes dans les musées de sciences en Californie ( $x \mid x^{\mathrm{e}}-\mathrm{xx}{ }^{\mathrm{e}}$ siècles) », Culture \& Musées, 30 | 2017, 111-131.

Référence électronique Leslie Madsen-Brooks, « « Je ne me suis pas perdue et ne souhaite pas que l'on me trouve ». Stratégies des femmes dans les musées de sciences en Californie (xIx ${ }^{\mathrm{e}-\mathrm{xx}} \mathrm{e}^{\mathrm{e}}$ siècles) », Culture \& Musées [En ligne], 30 | 2017, mis en ligne le 19 juin 2018, consulté le 16 juin 2021. URL : http://journals.openedition.org/culturemusees/1229; DOI :

https://doi.org/10.4000/culturemusees.1229

\section{Auteur}

\section{Leslie Madsen-Brooks}

Leslie Madsen-Brooks est professeure associée d'histoire et directrice de conception et d'évaluation pédagogique à l'université d'État de Boise. Elle étudie comment les gens qui n'ont été initialement formé en histoire ou en sciences ont participé à ces disciplines depuis le xIx ${ }^{\mathrm{e}}$ siècle jusqu'à l'âge des médias sociaux. Elle s'intéresse particulièrement à la manière dont les femmes ont façonné leur discours sur la botanique et l'horticulture en Californie.

Courriel : lesliemadsen-brooks[at]boisestate.edu

\section{Droits d'auteur}

Culture \& Musées 